\title{
THE PROBLEM OF MUTUALLY UNBIASED BASES IN DIMENSION 6
}

\author{
PHILIPPE JAMING, MÁTÉ MATOLCSI, AND PÉTER MÓRA
}

\begin{abstract}
We outline a discretization approach to determine the maximal number of mutually unbiased bases in dimension 6 . We describe the basic ideas and introduce the most important definitions to tackle this famous open problem which has been open for the last 10 years. Some preliminary results are also listed.
\end{abstract}

Dedicated to Prof. Warwick de Launey on the occasion of his 50th birthday

Keywords and phrases. Mutually unbiased bases, complex Hadamard matrices

\section{INTRODUCTION}

This paper is based on the talk given by the second author at the International Conference on Design Theory and Applications, NUI, Galway, July 1-3, 2009.

The notion of mutually unbiased bases (MUBs) constitutes a basic concept of Quantum Information Theory and plays an essential role in quantum-tomography [15, 23], quantum cryptography [4, 6, 20], the mean king problem [1] as well as in constructions of teleportation and dense coding schemes [22].

Recall that two orthonormal bases of $\mathbb{C}^{d}, \mathcal{A}=\left\{\mathbf{e}_{1}, \ldots, \mathbf{e}_{d}\right\}$ and $\mathcal{B}=$ $\left\{\mathbf{f}_{1}, \ldots, \mathbf{f}_{d}\right\}$ are said to be unbiased if, for every $1 \leq j, k \leq d,\left|\left\langle\mathbf{e}_{j}, \mathbf{f}_{k}\right\rangle\right|=$ $\frac{1}{\sqrt{d}}$. A set $\mathcal{B}_{0}, \ldots \mathcal{B}_{m}$ of orthonormal bases is said to be (pairwise) mutually unbiased if every two of them are unbiased. It is well-known ( see e.g. [2, 5, 23]) that the number of mutually unbiased bases (MUBs) in $\mathbb{C}^{d}$ cannot exceed $d+1$. It is also known that $d+1$ such bases can be constructed if the dimension $d$ is a prime or a prime power (see e.g. [2, 11, 12, 13, 15, 17, 23]). Such a set of $d+1$ MUBs in dimension $d$ is called a complete set. If the dimension $d=p_{1}^{\alpha_{1}} \ldots p_{k}^{\alpha_{k}}$ is composite

M. Matolcsi was supported by OTKA Grant No. K77748. 
then very little is known except for the fact that there are at least $p_{j}^{\alpha_{j}}+1$ mutually unbiased bases in $\mathbb{C}^{d}$ where $p_{j}^{\alpha_{j}}$ is the smallest of the prime-power divisors. Thus, the first case where the largest number of mutually unbiased bases is unknown is $d=6$ :

\section{Problem 1.1.}

What is the maximal number of pairwise mutually unbiased bases in $\mathbb{C}^{6}$ ?

Although this famous open problem has received considerable attention over the past few years $([5,7,6,16,19,21])$, it remains wide open. Since $6=2 \times 3$, we know that there are at least 3 mutually unbiased bases in $\mathbb{C}^{6}$ (see also [24, 16] for infinite families of MUB-triplets), but so far tentative numerical evidence [7, 8, 10, 24] suggests that there are no more than 3, a fact apparently first conjectured by Zauner [24].

\section{Conjecture 1.2.}

The maximal number of pairwise mutually unbiased bases in $\mathbb{C}^{6}$ is 3 .

One reason for the slow progress is that mutually unbiased bases are naturally related to complex Hadamard matrices. Indeed, if the bases $\mathcal{B}_{0}, \ldots, \mathcal{B}_{m}$ are mutually unbiased we may identify each $\mathcal{B}_{l}=$ $\left\{\mathbf{e}_{1}^{(l)}, \ldots, \mathbf{e}_{d}^{(l)}\right\}$ with the unitary matrix

$$
\left[H_{l}\right]_{k, j}=\left[\left\langle\mathbf{e}_{k}^{(l)}, \mathbf{e}_{j}^{(0)}\right\rangle_{1 \leq k, j \leq d}\right],
$$

i.e. the $k$-th row of $H_{l}$ consists of the coordinates of the $k$-th vector of $\mathcal{B}_{l}$ in the basis $\mathcal{B}_{0}$. (Throughout the paper the scalar product $\langle.,$. of $\mathbb{C}^{d}$ is linear in the first variable and conjugate-linear in the second. Note also that for convenience of computer programming we use the unconventional definition that the rows of the matrices correspond to the vectors of the bases.) With this convention, $H_{0}=I$ the identity matrix and all other matrices are unitary and have entries of modulus $1 / \sqrt{d}$. Therefore, the matrices $\sqrt{d} H_{l}$ have all entries of modulus 1 and complex orthogonal rows (and columns). Such matrices are called complex Hadamard matrices. It is clear that the existence of a family of mutually unbiased bases $\mathcal{B}_{0}, \ldots, \mathcal{B}_{m}$ is thus equivalent to the existence of a family of complex Hadamard matrices $\sqrt{d} H_{1}, \ldots, \sqrt{d} H_{m}$ such that for all $1 \leq j \neq k \leq m, \sqrt{d} H_{j} H_{k}^{*}$ is again a complex Hadamard matrix. In such a case we will say that these complex Hadamard matrices are mutually unbiased.

In particular, the existence of $4 \mathrm{MUBs} \mathcal{B}_{0}, \mathcal{B}_{1}, \mathcal{B}_{2}, \mathcal{B}_{3}$ in dimension 6 is equivalent to the existence of 3 mutually unbiased Hadamard matrices $H_{1}, H_{2}, H_{3}$. Therefore, in an attempt to prove that no collection of 4 
MUBs exist in dimension 6 it is enough to prove that no triplet of mutually unbiased Hadamard matrices exist. This will be the core of our argument in this note.

A complete classification of complex Hadamard matrices, however, is only available up to dimension 5 (see [14]) which allows for a complete classification of MUBs (see [9]). The classification in dimension 6 is still out of reach despite recent efforts [3, 19, 21]. This is one of the reasons for Problem 1.1 to be difficult.

In this paper we outline a discretization approach that is likely to lead to the proof of Conjecture 1.2 in the near future. Once all the ideas are properly implemented in a computer code, an exhaustive search will be carried out to prove Conjecture 1.2. We will include all the basic definitions and ideas as well as some preliminary results. We remark here that a partial result of this approach has already been completed: in [16] we assumed that the first Hadamard matrix $H_{1}$ comes from the two-parameter Fourier family of complex Hadamard matrices, and we proved by discretization and an exhaustive computer search that in such a case a MUB-quartet $I, H_{1}, H_{2}, H_{3}$ cannot exist. In this paper, however, we tackle the general case, so that we must consider $H_{1}$ as any complex Hadamard matrix of dimension 6. This complicates matters quite considerably as the number of cases to check after the discretization increases by orders of magnitude. As an optimistic note let us recall here that the non-existence of a projective plane of order 10 was also proved by an exhaustive computer search [18].

\section{Discretization}

The proof proceeds by contradiction, via a discretization scheme. Assume that there exists a collection of 4 MUB's in $\mathbb{C}^{6}$. Equivalently, there exist $6 \times 6$ complex Hadamard matrices $A, B, C$ having all entries of modulus 1 , such that the rows (and thus the columns) are complex orthogonal, and we have the unbiased condition: for any two rows $u, v$ coming from different matrices we have $|\langle u, v\rangle|=\sqrt{6}$. (Recall that for the purposes of this note the rows of the matrices correspond to the vectors of the bases.) In such a case the orthonormal bases $\frac{1}{\sqrt{6}} A, \frac{1}{\sqrt{6}} B, \frac{1}{\sqrt{6}} C$ accompanied with the identity matrix $I d$ correspond to a family of 4 MUB's. We assume that such matrices $A, B, C$ exist and try to reach a contradiction.

After multiplying rows and columns by appropriate scalars if necessary, we can assume that all coordinates of the first row and column of $A$ are 1's, and all coordinates of the first column of all other matrices are 
1 's (i.e. we assume that all vectors in the bases $A, B, C$ have first coordinate 1 , and the first vector in basis $A$ is an all 1 's vector). All the other coordinates in the matrices are complex numbers of modulus 1, i.e. they are of the form $e^{2 \pi i \rho}$ with $\rho \in[0,1)$. We will use a discretization approach. Let $N$ be a positive integer, called the discretization parameter. We partition the interval $[0,1)$ into $N$ sub-intervals $I_{0}^{(N)}, I_{1}^{(N)}, \ldots, I_{N-1}^{(N)}$ of equal length, i.e. $I_{j}^{(N)}=[j / N,(j+1) / N)$. (Other partitions are also possible, but this seems most convenient for programming.) Now, any entry $e^{2 \pi i \rho}$ in any of the matrices $A, B, C$ will be represented by the integer $j$ if $\rho \in I_{j}^{(N)}$ (note that $0 \leq j \leq N-1$ ). This means: whenever we see an entry $j$ somewhere in a matrix then we conclude that the original phase $\rho$ must lie somewhere in the interval $I_{j}^{(N)}$. We have no more and no less information than this. We also agree that the first coordinate of each row will be represented by 0 , keeping in mind that it represents exactly 1 , without error (and not the interval $I_{0}^{(N)}$ ).

In short: we will exclusively be dealing with row vectors of the form

$$
u=\left(0, j_{1}, j_{2}, j_{3}, j_{4}, j_{5}\right)
$$

where $0 \leq j_{k} \leq N-1$ and the first coordinate 0 represents 1 without error, while the other coordinates $j_{k}$ mean that the actual entry $\rho_{k}$ falls into the interval $I_{j_{k}}^{(N)}$. In notation, the original matrix will be denoted by $A$, while its representative integer matrix will be denoted by $\tilde{A}$. The entries of $A$ will be denoted by $\rho_{m, k}$, while those of $\tilde{A}$ will be denoted by $j_{m, k}$.

There are altogether $N^{5}$ vectors of the form (1).

Also, there is a natural ordering among these vectors: $u \leq v$ if and only if it is so in lexicographical order. We will use this ordering throughout this note.

\section{The SEARCH FOR THE DISCRETIZED HADAMARD MATRIX $\tilde{A}$}

The matrix $\tilde{A}$ is an integer matrix with first row and column consisting of 0 's and the core of the matrix containing integers between 0 and $N-1$. We introduce the following definition:

Definition 3.1. Given an integer matrix $\tilde{A}$ with first row and column consisting of 0 's and the core of the matrix containing integers $j_{m, k}$ between 0 and $N-1$, we will say that $\tilde{A}$ is an $N$-discretized representative of a complex Hadamard matrix if there exists a complex Hadamard matrix $A$ with entries $\rho_{m, k}$ such that $\rho_{m, k} \in I_{j_{m, k}}$. In notation $\tilde{A} \in H A D_{N}$, 
where $H A D_{N}$ denotes the set of $N$-discretized representatives of complex Hadamard matrices.

The aim of this section is to describe an algorithm to efficiently search for all possible matrices $\tilde{A} \in H A D_{N}$. Upon strong numerical evidence [21], it is conjectured that the manifold of $6 \times 6$ complex Hadamard matrices is 4-dimensional. Therefore we expect that the cardinality of $H A D_{N}$ will be approximately $c N^{4}$ for some constant $c$. Nevertheless, the task of finding all possible $\tilde{A}$ is daunting at first glance. There are $N^{25}$ possible $N$-discretized matrices altogether, and we must select the ones belonging to $H A D_{N}$. The number $N^{25}$ is of course astronomical even for $N \approx 50$, but we will see that with an intelligent approach the task can still be carried out.

There are a few properties we can assume about $\tilde{A}$ without loss of generality. We already assumed that the first row and column consist of 0's. We can also assume that both the rows and columns are arranged so that they increase with respect to lexicographical order. This can be arranged by repeated permutation of rows and columns. (This is not entirely trivial because ordering the rows lexicographically can actually spoil such an ordering of the columns and vice versa. However, if one writes out the matrix entries row-after-row in one 36-long row vector, then it is clear that this vector will decrease lexicographically irrespectively of whether you make an ordering of the rows or the columns. Therefore such a repeated ordering of rows and columns will terminate in finite steps, and will produce a matrix such that both the rows and the columns increase in lexicographical order.) This automatically implies that the entries of the second row and second column are both monotonically increasing. This is a very convenient property, because it restricts the possibilities for the second row and column quite strongly.

We can also assume that the second row is less than or equal to the second column in lexicographical order (this can be arranged by transposition of $\tilde{A}$ if necessary).

We must make use of the fact that the rows (and columns) of $A$ are complex orthogonal to each other. The first row and column of $\tilde{A}$ consist of 0 's (representing the entry 1 in $A$, without error). Therefore, we have 5 unknown rows and columns of $\tilde{A}$. All of these rows and columns have the form (1). The orthogonality condition with the first row (and column) makes it natural to introduce the following definition:

Definition 3.2. We will say that a vector $u$ of the form (1) belongs to $O R T_{N}$ if there exist $\phi_{k} \in I_{j_{k}}$ such that $1+\sum_{k=1}^{5} e^{2 i \pi \phi_{k}}=0$. 
Note that $O R T_{N}$ is a "small" subset of all the vectors of form (1), containing only those vectors which represent vectors being orthogonal to the vector $(1,1,1,1,1,1)$. Clearly, all rows and columns of $\tilde{A}$ must belong to $O R T_{N}$. Therefore it is very important to determine the set $O R T_{N}$ as precisely as we can. We achieve this by the following "check the descendants" method.

Let $u=\left(0, j_{1}, j_{2}, j_{3}, j_{4}, j_{5}\right)$, and let $r_{j_{k}}$ denote the midpoint of the interval $I_{j_{k}}$ (the superscript $N$ has been dropped from the notation for convenience). If $u \in O R T_{N}$ then the trivial error bound (see Lemma 3.1 in [16]) gives

$$
\left|1+\sum_{k=1}^{5} e^{2 \pi i r_{j_{k}}}\right| \leq \frac{5 \pi}{N} .
$$

This is too crude, but we can iterate it to the "children" of $u$. Namely, assume that the numbers $\phi_{k}$ exist as in Definition 3.2, For each interval $I_{j_{k}}$ the value $\phi_{k}$ must lie in either the left or the right half of $I_{j_{k}}$. There are 32 choices, according to whether we consider the left or the right half of each interval $I_{j_{k}}$. These choices are called the 32 "children" of $u$. Clearly, at least one of these children needs to satisfy (2) with $\frac{5 \pi}{2 N}$ on the right hand side (and its own midpoints substituted to the left hand side, of course, instead of $r_{j_{k}}$ ). If none of the children satisfy this, then $u$ can be discarded. Of course we iterate this to grandchildren, and so on, down to 7-8 generations. The vector $u$ survives this test if it has at least one surviving descendant in each generation.

Remark 3.1. The set $O R T_{N}$ is clearly invariant under permutations of the last 5 coordinates $j_{1}, j_{2}, j_{3}, j_{4}, j_{5}$. Therefore it makes sense to introduce the set $O R T_{N, \text { mon }}$ of vectors in $O R T_{N}$ with monotonically increasing coordinates. To save time, in the actual computer code we first find the vectors of $O R T_{N, m o n}$ by the method above, and then we permute the last 5 coordinates to arrive at the set $O R T_{N}$.

Remark 3.2. There exists also an improved error bound (see Lemma 3.2 in [16]). It is somewhat slower to check by computer and it is reasonable to believe that we arrive at the same set $O R T_{N}$ by applying either error bounds.

Remark 3.3. We have implemented a computer code for selecting the set $O R T_{N}$. For example, for $N=17$ we have $\left|O R T_{N}\right|=58450$, for $N=19,\left|O R T_{N}\right|=82630$, and for $N=53,\left|O R T_{N}\right|=1875110$. Experience shows that the set $O R T_{N}$ is unexpectedly large if $N$ is divisible by 2 or 3 . Therefore, we have mainly restricted our attention to $N$ being a prime. 
Remark 3.4. The optimal choice of $N$ seems to be crucial for the success of the project. Clearly, if $N$ is too small then the error bounds are not good enough and we will not reach a contradiction in the forthcoming steps (see Section 4 below). However, if $N$ is too large then the size of the sets $O R T_{N}$ and correspondingly $H A D_{N}$ will be far too large to be manageable. At present we believe that the optimal choice of $N$ is around $N \approx 50$.

Let us turn back to the construction of $\tilde{A}$. All rows and columns must come from $O R T_{N}$, and they must be pairwise $N$ - orthogonal in the following sense:

Definition 3.3. We will say that the vectors $u=\left(0, j_{1}, j_{2}, j_{3}, j_{4}, j_{5}\right)$ and $v=\left(0, m_{1}, m_{2}, m_{3}, m_{4}, m_{5}\right)$ are $N$-orthogonal if there exist numbers $\phi_{k}$ and $\psi_{k}$ in the intervals $I_{j_{k}}$ and $I_{m_{k}}$, such that $1+\sum_{k=1}^{5} e^{2 i \pi\left(\phi_{k}-\psi_{k}\right)}=0$.

This property is clearly shift-invariant in the sense that it only depends on the values $\left(j_{1}-m_{1}, \ldots j_{5}-m_{5}\right)$ modulo $N$. We can therefore take $m_{1}=\cdots=m_{5}=0$ and correspondingly $v_{0}=(0,0 \ldots, 0)$, (where the last 5 coordinates represent the interval $I_{0}$, of course) and define the set $O R T_{e p s, N}$ as the set of vectors of the form (1) which are $N$ orthogonal to $v_{0}$. (The notation $O R T_{e p s, N}$ indicates that the vector $v_{0}$ contains an "epsilon" of error, because the last 5 coordinates represent the interval $I_{0}$ and not the exact number 1.) With this notation the shift-invariance means that $u$ and $v$ will be $N$-orthogonal if and only if the vector $\left(j_{1}-m_{1}, \ldots j_{5}-m_{5}\right)(\bmod N)$ is in $O R T_{e p s, N}$.

Having constructed the set $O R T_{N}$ previously, it is now easy to obtain $O R T_{e p s, N}$. Indeed, by definition a vector $u=\left(0, j_{1}, \ldots j_{5}\right)$ can only be $N$-orthogonal to $v_{0}$ if there exist numbers $\phi_{k}$ in the intervals $I_{j_{k}}$ and $\psi_{k}$ in $\left[0, \frac{1}{N}\right)$, such that $1+\sum_{k=1}^{5} e^{2 i \pi\left(\phi_{k}-\psi_{k}\right)}=0$. But then the numbers $\phi_{k}-\psi_{k}$ must fall in the intervals $I_{j_{k}-\epsilon_{k}}$ where $\epsilon_{k}$ is either 0 or 1 , and hence the vector $u_{\epsilon}=\left(0, j_{1}-\epsilon_{1}, \ldots, j_{5}-\epsilon_{5}\right)$ is in $O R T_{N}$.

Therefore, $O R T_{e p s, N}$ will consist of all the vectors of the form $u^{\epsilon}=$ $\left(0, j_{1}+\epsilon_{1}, \ldots, j_{5}+\epsilon_{5}\right)$, where $\epsilon_{k}$ is 0 or 1 , and the vector $\left(0, j_{1}, \ldots, j_{5}\right)$ is in $O R T_{N}$.

Remark 3.5. Each $u \in O R T_{N}$ gives rise to 32 different $u^{\epsilon}$ above. One could therefore expect that the size of $O R T_{\text {eps }, N}$ will be nearly 32 times the size of $O R T_{N}$. This is not so, however, because there will be many coincidences. Experience shows that the size of $O R T_{e p s, N}$ is approximately 4 times the size of $O R T_{N}$, regardless of the value of $N$. 
Now we are ready to conduct a search for the possible matrices $\tilde{A}$. The first row and column are full of 0's. We then build up the matrix with a row-by-column approach. We fit in the second row, then the second column, then the third row, then the third column, etc. At each step we must consider that:

- each row and column must come from $O R T_{N}$.

- each row (resp. column) must be lexicographically larger than any previous rows (resp. columns). In particular, the entries of the second row and column are monotonically increasing, i.e. they belong to $O R T_{N, m o n}$.

- the second column must be lexicographically larger than or equal to the second row.

- each row (resp. column) must be $N$-orthogonal to any previous rows (resp. columns). This is equivalent to the fact that the pairwise differences of the rows (resp. columns) modulo $N$ must be contained in $O R T_{\text {eps }, N}$.

- each row (resp. column) must be compatible with the already existing entries of the matrix (e.g. when we fit in the fourth row, then its first 3 coordinates are already fixed because the first three columns of the matrix have already been filled out previously).

We have implemented a computer code which executes the search as described above. The running time is still reasonable, within 1-4 days, depending on $N$. However, the number of selected matrices $\tilde{A}$ is unexpectedly large. It is in the range of $10^{9}-5 \cdot 10^{10}$ as $N$ ranges from 17 to 53. Let $P R E H A D_{N}$ denote the set of matrices obtained by this search. Clearly, $H A D_{N} \subset P R E H A D_{N}$.

Have we made all possible restrictions so as to list exclusively the matrices $\tilde{A}$ belonging to $H A D_{N}$ ? In other words, is it true that $H A D_{N}=P R E H A D_{N}$ ? It turns out that this is not the case, and there is an important possibility for further pruning. Consider a matrix $\tilde{A} \in P R E H A D_{N}$. There are 25 non-trivial entries in $\tilde{A}$ (the first row and column being trivial), all of which represent intervals $I_{j_{m, k}}$ of length $1 / N$. Once again we can "check the descendants" of $\tilde{A}$. That is, we can take left or right halves of each 25 intervals $I_{j_{m, k}}$, and therefore consider the $2^{25}$ children of $\tilde{A}$. Obviously, at least one of these children need to satisfy stricter pairwise orthogonality conditions of rows and columns. If none of the children do, then $\tilde{A}$ can be discarded, i.e. it does not belong to $H A D_{N}$. Of course, checking $2^{25}$ children is very slow, but if one proceeds row-by-row then only a few thousand children 
need to be actually checked. We have not rigorously implemented this step in our computer code. Nevertheless, preliminary results suggest that only a small fraction of the matrices in $P R E H A D_{N}$ will pass this test, i.e. $H A D_{N}$ will be significantly smaller in size than $P R E H A D_{N}$. This is very important for the running time of the overall algorithm, as the size of $H A D_{N}$ should definitely be kept in the range $10^{8}-10^{9}$ even for $N \approx 50$.

\section{Stage 2: vectors unbiased to $\tilde{A}$, And Reaching A CONTRADICTION}

Let us fix a matrix $\tilde{A} \in H A D_{N}$. We want to prove that the pair $(I d, \tilde{A})$ cannot be extended by matrices $\tilde{B}, \tilde{C}$ so as to meet all orthogonality and unbiasedness conditions. The rows of $\tilde{B}$ and $\tilde{C}$ are of the form (11) and they must be "unbiased" to all six rows of $\tilde{A}$. Therefore, as a next step, we must obtain a list of all such vectors.

Remark 4.1. We are actually free to use a different discretization parameter $N^{\prime}$ for the matrices $\tilde{B}$ and $\tilde{C}$. It may well reduce the running time if we use optimal choices for $N$ and $N^{\prime}$. Experience shows (see [16]) that it makes sense to choose $N^{\prime}$ considerably smaller than $N$. However, for the sake of simplicity we will keep $N=N^{\prime}$ throughout this note.

As the first row of $\tilde{A}$ is invariably $(0,0,0,0,0,0)$ (representing 1 's in the first row of $A$, without error) it makes sense to introduce the following definition:

Definition 4.1. We will say that a vector $u=\left(0, j_{1}, j_{2}, j_{3}, j_{4}, j_{5}\right)$ belongs to the set $U B_{N}$ if there exist $\phi_{k} \in I_{j_{k}}$ such that $\left|1+\sum_{k=1}^{5} e^{2 i \pi \phi_{k}}\right|=$ $\sqrt{6}$. We will say that $u$ belongs to $U B_{N, \text { mon }}$ if the coordinates of $u$ are monotonically increasing.

The set $U B_{N}$ can be constructed in a similar way as $O R T_{N}$. With $r_{j_{k}}$ denoting the midpoint of the interval $I_{j_{k}}$ the trivial estimate gives

$$
|| 1+\sum_{k=1}^{5} e^{2 i \pi r_{j_{k}}}|-\sqrt{6}| \leq \frac{5 \pi}{N} .
$$

This is too crude, of course, and the descendants of $u$ need to be checked for some 7-8 generations.

Remark 4.2. Once again, the set $U B_{N}$ is invariant under the permutation of the last 5 coordinates $j_{1}, j_{2}, j_{3}, j_{4}, j_{5}$. Therefore, in practice, we first check monotonically increasing vectors only, and obtain $U B_{N, m o n}$. Then we permute the coordinates to obtain $U B_{N}$. 
Remark 4.3. The set $U B_{N}$ is much larger than $O R T_{N}$. This can be expected because orthogonality of complex vectors induces two conditions (the real part and imaginary part both being zero) while unbiasedness only induces one condition.

Remark 4.4. We have implemented a code for listing the set of vectors $U B_{N}$. For example, for $N=17$ we have $\left|U B_{N}\right|=479340$, while for $N=19,\left|U B_{N}\right|=764060$.

We will also need a set $U B_{e p s, N}$ which is analogous to $O R T_{e p s, N}$.

Definition 4.2. We will say that the vectors $u=\left(0, j_{1}, j_{2}, j_{3}, j_{4}, j_{5}\right)$ and $v=\left(0, m_{1}, m_{2}, m_{3}, m_{4}, m_{5}\right)$ are $N$-unbiased if there exist numbers $\phi_{k}$ and $\psi_{k}$ in the intervals $I_{j_{k}}$ and $I_{m_{k}}$, such that $\left|1+\sum_{k=1}^{5} e^{2 i \pi\left(\phi_{k}-\psi_{k}\right)}\right|=$ $\sqrt{6}$.

This property is again shift-invariant in the sense that it only depends on the values $\left(j_{1}-m_{1}, \ldots j_{5}-m_{5}\right)$ modulo $N$. We can therefore take $m_{1}=\cdots=m_{5}=0$ and correspondingly $v_{0}=(0,0 \ldots, 0)$, (where the last 5 coordinates represent the interval $I_{0}$, of course) and define the set $U B_{e p s, N}$ as the set of vectors of the form (1) which are $N$-unbiased to $v_{0}$. With this notation the shift-invariance means that $u$ and $v$ will be $N$-unbiased if and only if the vector $\left(j_{1}-m_{1}, \ldots j_{5}-m_{5}\right)(\bmod N)$ is in $U B_{\text {eps }, N}$.

Let the vector $v$ of the form (1) be any row of either $\tilde{B}$ or $\tilde{C}$. As the first row of $\tilde{A}$ is invariably $(0,0,0,0,0,0)$ (representing the 1 's in the first row of $A$, without error), we conclude that $v \in U B_{N}$. Let $a_{2}, \ldots a_{6}$ denote the last five rows of $\tilde{A}$. Then, by definition, the differences $v-a_{j}$ modulo $N$ must belong to $U B_{e p s, N}$ for all $j=2, \ldots 6$. Let $U B_{\tilde{A}}$ denote the set of vectors $v$ which satisfy these conditions. The notation $U B_{\tilde{A}}$ reflects that these are the vectors which are "unbiased" to all rows of $\tilde{A}$. By what has been said above, all rows of $\tilde{B}$ and $\tilde{C}$ must belong to $U B_{\tilde{A}}$.

Remark 4.5. We have implemented a code to obtain the set $U B_{\tilde{A}}$. Experience shows that the size of $U B_{\tilde{A}}$ is largely independent of the choice of $\tilde{A}$, and it has between $10^{3}-10^{4}$ vectors as $N$ ranges from 17 to 53 .

Having constructed $U B_{\tilde{A}}$ we must show that $\tilde{B}$ and $\tilde{C}$ cannot be built from these vectors satisfying all orthogonality and unbiased conditions.

Consider the vectors in $U B_{\tilde{A}}$ and try to build the matrix $\tilde{B}$ out of them. This means that we need to find 6 vectors $b_{1}, \ldots b_{6}$ such that the pairwise differences $b_{k}-b_{m}$ modulo $N$ all belong to $O R T_{e p s, N}$. Counting 
constraints and parameters one would expect that only a finite number of triplets of MUB's $(I d, A, B)$ exists, and a MUB-pair $(I d, A)$ can generically not be extended to a triplet $(I d, A, B)$. This would give us hope that a contradiction is reached most of the times while trying to build $\tilde{B}$. However, recent results [16, 8] show that infinite families of MUB-triplets do exist. Numerical practice also shows that the matrix $\tilde{B}$ can indeed be built from the vectors of $U B_{\tilde{A}}$ for all $\tilde{A}$. Therefore, we do not get an immediate contradiction. Instead, for each $\tilde{B}$ we must go on and select the vectors $U B_{\tilde{A}, \tilde{B}}$ which are unbiased to all rows of $\tilde{A}$ and $\tilde{B}$, and we must try to build a matrix $\tilde{C}$ out of the vectors $U B_{\tilde{A}, \tilde{B}}$. The contradiction is reached only at this point. That is, if $N$ is large enough the matrix $\tilde{C}$ cannot be constructed from $U B_{\tilde{A}, \tilde{B}}$ to meet all orthogonality conditions. Experience shows that $N$ must be larger than 30 to reach a contradiction. This part of the project is currently under implementation. It would be desirable to reach a contradiction for each $\tilde{A}$ within a few seconds of computing time.

For the overall success of the project two tasks need to be considered in the near future. One is the implementation of the ideas of the last paragraph of Section 3 to bring down the number of possible $\tilde{A}$ 's to the region $10^{8}-10^{9}$. The other is to reach a contradiction for each $\tilde{A}$ within a few seconds of computing time by not being able to construct $\tilde{B}$ and $\tilde{C}$. A nice feature of the overall project is that once the algorithm is completed, it is very easy to distribute the calculations among several hundreds of computers, and thus reducing the running time by $2-3$ orders of magnitude.

Finally, we remark that the entire discretization procedure described above has already been completed in [16] in the restricted setting when $A$ is assumed to belong to the Fourier family $F(a, b)$ of complex Hadamard matrices.

Theorem 4.6. [Theorem 1.4 in [16]]

None of the pairs $(I d, F(a, b))$ of mutually unbiased orthonormal bases can be extended to a quartet $(I d, F(a, b), B, C)$ of mutually unbiased orthonormal bases.

In that case we used the discretization parameters $N=180$ for $\tilde{A}$ and $N^{\prime}=19$ for $\tilde{B}$ and $\tilde{C}$. Due to some well-known equivalence relations only a few hundred possible discretized matrices $\tilde{A}$ needed to be considered, and a contradiction was quickly reached for all of them. The documentation of that search is available at [25]. The difficulty in the general case is that the number of matrices $\tilde{A}$ becomes very large 
if $N$ is chosen large, while if $N$ is small then a contradiction is reached very slowly (or not reached at all!) in the second stage of the search.

\section{REFERENCES}

[1] Y. Aharonov \& B.-G. Englert, The mean king's problem: Spin 1. Z. Naturforsch. 56a, (2001) 16.

[2] S. Bandyopadhyay, P. O. Boykin, V. Roychowdhury \& F. Vatan $A$ New Proof for the Existence of Mutually Unbiased Bases. Algorithmica 34 (2002), 512-528.

[3] K. Beauchamp \& R. Nicoara, Orthogonal maximal Abelian *-subalgebras of the $6 \times 6$ matrices. Linear Algebra Appl. 428 (2008), 1833-1853.

[4] H. Bechmann-Pasquinucci \& W. Tittel, Quantum cryptography using larger alphabets. Phys. Rev. A, 61 (2000), no. 6, 062308, 6 pp.

[5] I. Bengtsson, W. Bruzda, A. Ericsson, J.-A. Larsson, W. Tadej \& K. ŻYCZKOwski, Mutually unbiased bases and Hadamard matrices of order six. J. Math. Phys. 48 (2007), no. 5, 052106, 21 pp.

[6] C. H. Bennett \& G. Brassard, Quantum cryptography: Public key distribution and coin tossing. In Proceedings of the IEEE Intl. Conf. Computers, Systems, and Signal Processing, pages 175-179. IEEE, 1984.

[7] S. Brierley \& S. WeigerT, Maximal sets of mutually unbiased quantum states in dimension six. arXiv:0808.1614 (quant-ph).

[8] S. Brierley \& S. Weigert, Constructing Mutually Unbiased Bases in Dimension Six. arXiv:0901.4051 (2009)

[9] S. Brierley, S. Weigert \& I. Bengtsson, All Mutually Unbiased Bases in Dimensions Two to Five arXiv:0907.4097 (2009)

[10] P. Butterley \& W. Hall Numerical evidence for the maximum number of mutually unbiased bases in dimension six. Physics Letters A 369 (2007) 5-8.

[11] M. Combescure The mutually unbiased bases revisited. Adventures in mathematical physics, 29-43, Contemp. Math., 447, Amer. Math. Soc., Providence, RI, 2007.

[12] M. Combescure Circulant matrices, Gauss sums and mutually unbiased bases I. The prime number case. Available at arXiv:0710.5642 1 .

[13] M. Combescure Circulant matrices, Gauss sums and mutually unbiased bases II. The prime power case. Available at arXiv:0710.5643 1 .

[14] U. HAAGERUP, Ortogonal maximal Abelian $*$-subalgebras of $n \times n$ matrices and cyclic $n$-roots. Operator Algebras and Quantum Field Theory (Rome), Cambridge, MA International Press, (1996), 296-322.

[15] I. D. Ivanovic, Geometrical description of quantal state determination. J. Phys. A 14 (1981), 3241.

[16] Ph. Jaming, M. Matolcsi, P. Móra, F. Szöllősi, M. Weiner, A generalized Pauli problem and an infinite family of MUB-triplets in dimension 6. J. Physics A: Mathematical and Theoretical, Vol. 42, Number 24, 245305, 2009.

[17] A. Klappenecker \& M. RÖtTeler Constructions of Mutually Unbiased Bases. Finite fields and applications, 137-144, Lecture Notes in Comput. Sci., 2948, Springer, Berlin, 2004.

[18] C.W.H. Lam, L. H. Thiel \& S. Swiercz, The non-existence of finite projective planes of order 10. Can. J. Math., Vol: XLI, (1989) 1117-1123. 
[19] M. Matolcsi, F. SzÖllősi, Towards a classification of $6 x 6$ complex Hadamard matrices. Open Systems \& Information Dynamics, 15, Issue:2, (June 2008), 93-108.

[20] J. M. RENES, Equiangular spherical codes in quantum cryptography. Quantum Inf. Comput. 5 (2005), 81-92.

[21] A. J. Skinner, V. A. Newell, R. Sanchez, Unbiased bases (Hadamards) for 6-level systems: Four ways from Fourier. arXiv:0810.1761 (2008)

[22] R. F. Werner, All teleportation and dense coding schemes. Quantum information and computation. J. Phys. A, 34 (2001), 7081-7094.

[23] W. K. Wootters \& B. D. Fields, Optimal state-determination by mutually unbiased measurements. Ann. Physics 191 (1989), 363-381.

[24] G. ZAUner, Quantendesigns Grundzüge einer nichtkommutativen Designtheorie. PhD thesis, Universität Wien, 1999. (available at http://www.mat.univie.ac.at/ neum/ms/zauner.pdf)

[25] Documentation of the results of [16]: http://www.math.bme.hu/ matolcsi/angpubl.html

P.J.: Université d'Orléans, Faculté des Sciences, MAPMO - FÉdération Denis Poisson, BP 6759, F 45067 Orléans Cedex 2, France

E-mail address: philippe.jaming@univ-orleans.fr

M. M.: Alfréd Rényi Institute of Mathematics, Hungarian Academy of Sciences POB 127 H-1364 Budapest, Hungary Tel: (+361) 483-8302,

FAX: (+361) 483-8333

E-mail address: matomate@renyi.hu

P. M. (and M. M. part time): BMe Department of Analysis, Egry J.

U. 1, H-1111 Budapest, Hungary

E-mail address: morapeter@gmail.com 